\title{
Kounis Syndrome (Type I) Following Bee Sting
}

\author{
Dr. S. Sivakumar ${ }^{1}$ M.D., Dr. N. Kirubanand ${ }^{2}$ M.D., Dr. R. Kannan ${ }^{3}$ D.M., \\ ${ }^{1}$ Associate Professor, Department of General Medicine, Government Thiruvannamalai \\ Medical College and Hospital \\ ${ }^{2}$ Assistant Professor, Department of General Medicine, Government Thiruvannamalai \\ Medical College and Hospital \\ ${ }^{3}$ Senior Resident, Department of General Medicine, Government Thiruvannamalai \\ Medical College and Hospital
}

\begin{abstract}
Bee stings are commonly encountered worldwide. Various manifestations after bee sting have been described ${ }^{1}$. Local reactions are common. Unusually, manifestations like vomiting, diarrhoea, dyspnoea, generalised oedema, acute renal failure, hypotension and collapse may occur. Rarely vasculitis, serum sickness, neuritis and encephalitis have been described which generally develop days to weeks after a sting. Acute coronary syndromes after hymenoptera stings and other environmental exposures are referred to as the Kounis syndrome or allergic myocardial ischaemia and infarction. The three variants of Kounis syndrome are Type I variant: includes patients with normal coronary arteries without predisposing factors for coronary artery disease in whom the acute release of inflammatory mediators can induce either coronary artery spasm without increase of cardiac enzymes and troponins or coronary artery spasm progressing to acute myocardial infarction with raised cardiac enzymes and troponins.

Type II variant: includes patients with culprit but quiescent pre-existing atheromatous disease in whom the acute release of inflammatory mediators can induce either coronary artery spasm with normal cardiac enzymes and troponins or plaque erosion or rupture manifesting as acute myocardial infarction.

Type III variant: includes patients with coronary thrombosis (including stent thrombosis) in whom aspirated thrombus specimens stained with hematoxylin-eosin and Giemsa demonstrate the presence of eosinophils and mast cells respectively. We report a 23 year old male who developed myocardial infarction after multiple bee stings over his body.
\end{abstract}

\section{Introduction}

We report a case of anterior wall myocardial infarction in a young man following a bee sting. Coronary angiography, performed following the acute phase, showed angiographically healthy coronary arteries.

\section{Case report}

A 23 year old male, Tutor in a private Polytechnic college was bitten by multiple bees over head and Chest region in the morning on 12/06/2016. On presentation vitals were stable. He was treated with intravenous antihistamines and antiemetics at a local private hospital. On 12/06/2016 evening 6 p.m. patient had developed severe chest pain, palpitation, dizziness and he was referred to Government Thiruvannamalai medical college. Past medical and surgical history was insignificant. ECG was taken which revealed anterior wall myocardial infarction (Fig 1). Subsequently CPK-MB, Troponin I were done which were found to be elevated (Fig 2). Echocardiography (Fig 3) was immediately done which revealed, regional wall motion abnormality in the Apex. Patient's serum homocysteine, lupus anticoagulant and anticardiolipin antibodies were within normal limits. Patient was treated with anticoagulants, anti-platelet drugs. Coronary angiography performed following the acute phase, showed angiographically healthy coronary arteries (Fig 4).

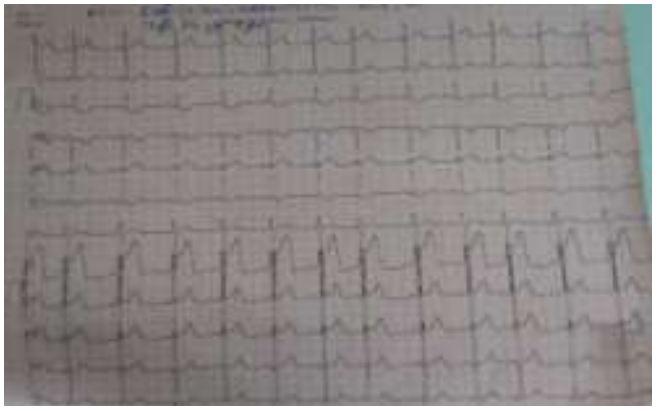

Fig 1: Initial ECG showing ST elevation

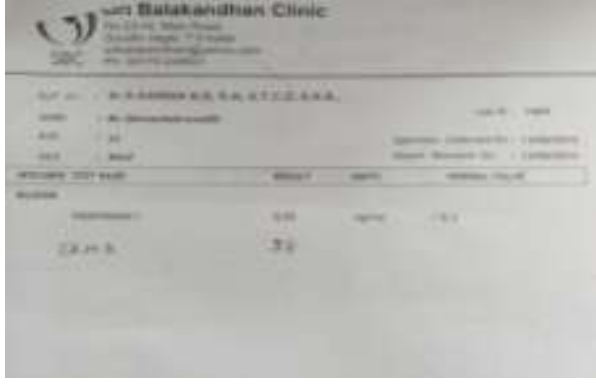

Fig 2: Elevated cardiac enzymes 
in Anterior Leads

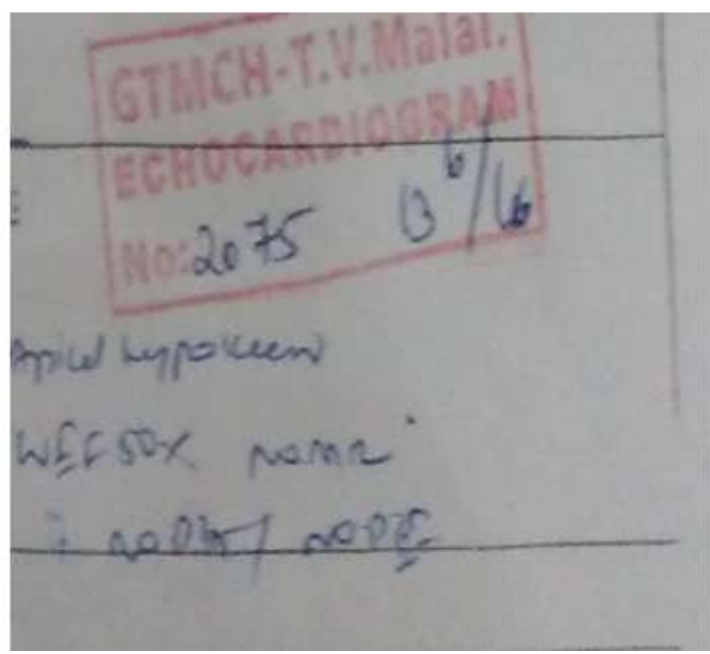

Fig 3: Initial Echo report showing Apical hypokinesia

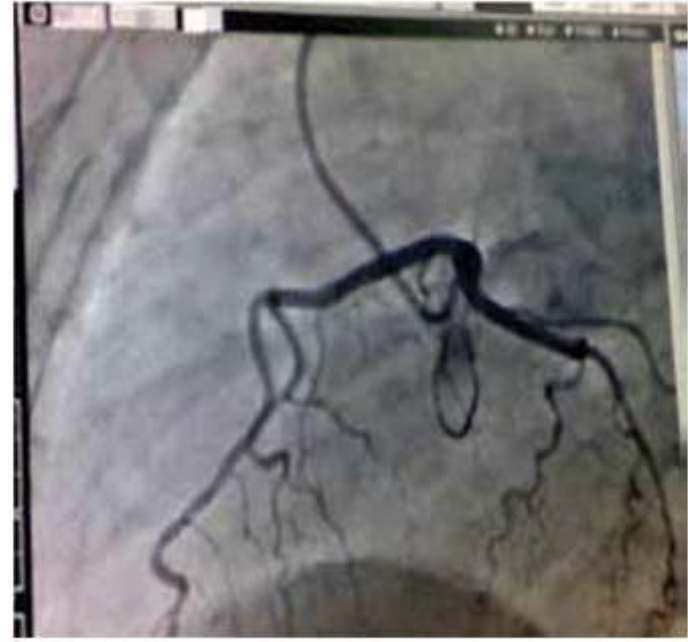

Fig 4: Normal Coronary Angiography

The subsequent ECG's which were done revealed return of the ST segment to normalcy (Fig 5). Repeat Echo on 14/06/2016 revealed no regional wall motion abnormalities (Fig 6). Patient's general condition improved and he was discharged. During Follow-up, the patient was subjected to stress ECG tread mill test which was found to be normal and hence lessening the chances of microvascular disease in our patient ${ }^{2}$

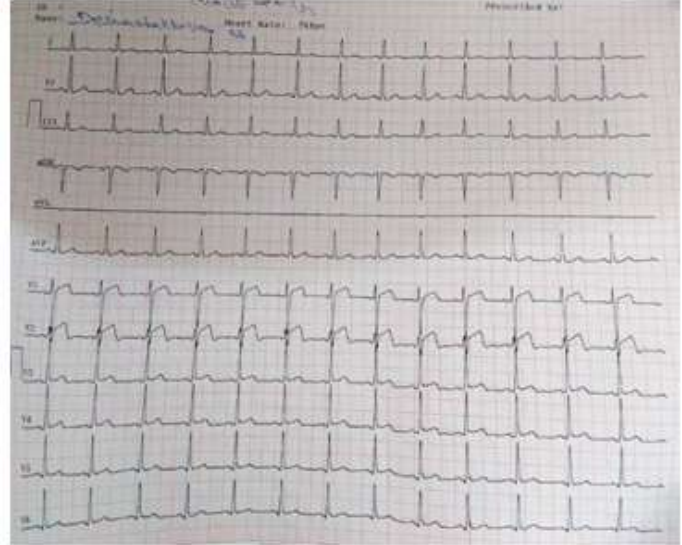

Fig 5: Follow-up Normal ECG

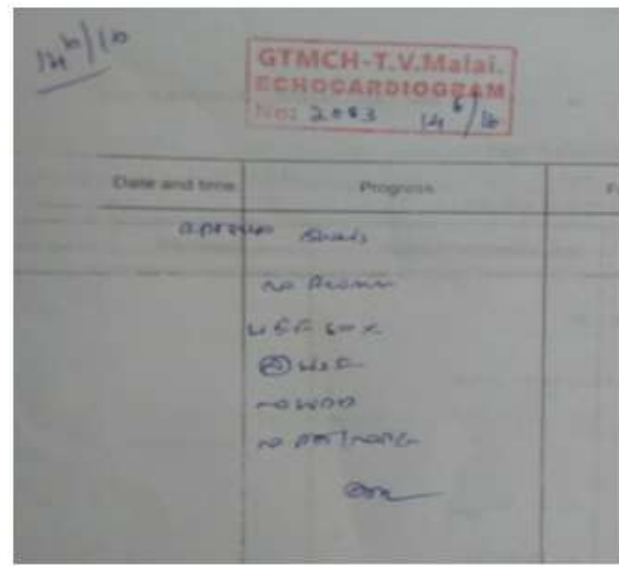

Fig 6: Follow-up Normal Echo report

\section{Discussion}

Cardiac complications rarely accompany bee sting. These may be serious and often fatal, being represented by cardiac arrhythmias, electrocardiographic abnormalities and conduction defects, as well as myocardial infarction, a rarely reported complication of bee sting. The extent and pathogenesis of cardiac toxicity from bee sting is not yet clearly defined. In literature we had few cases of myocardial infarction occurring after bee sting. Massing $\mathrm{JL}$ et $\mathrm{al}^{3}$ reported a case of inferior myocardial infarction in a young man following bee sting. Coronary angiography performed following the acute phase, showed angiographically healthy coronary arteries and minimal sequelae of myocardial necrosis in the inferior territory. The patient had gradually recovered. Mathieu Valla et $\mathrm{al}^{4}$ also reported myocardial infarction in a 45 -year-old man following an anaphylactic reaction to a wasp sting. Ceyhan et $\mathrm{al}^{5}$ reported a patient envenomed by a bee sting that caused myocardial damage compatible with non-ST elevation acute myocardial infarction. She developed a typical course of myocardial infarction; the ECG changes were reversed to almost normal limits. She had normal coronary angiography and reversible wall motion abnormalities. Myocardial damage following prolonged spasm in the coronary arteries may be the underlying factor. Harold D Levine ${ }^{6}$ reported two male patients who developed myocardial infarction after bee sting one aged 39, the other 66, each without previous overt heart disease. Each went into shock rapidly, after an interval of over a half-hour, developed chest pain and later, 
sequential electrocardiographic changes diagnostic of acute myocardial infarction. Each survived and had normal electrocardiograms before the sting. Though pre-existent coronary artery disease can be excluded in neither, the view is favoured that acute myocardial infarction in each patient was caused by deficient coronary perfusion secondary to anaphylactic shock induced by the wasp stings. Harold D. Levine et al also reported a 62-year-old man with previous angina who developed pulmonary oedema but no chest pain following wasp sting and went on to show rapidly reversed electrocardiographic changes attributable to subendocardial ischaemia or infarction. In a sense, this sequence fills the gap as an intermediate phase between the normal and the two individuals described here who developed pain after anaphylactic shock, then proceeded, perhaps through this phase, to develop transmural infarction. Sofiene Rekik $\mathrm{MD}^{7}$ et al reported a case of a 58- year-old man with transient inferior ST-segment elevation consistent with myocardial ischaemia after a single wasp sting. Urgent cardiac catheterization revealed normal coronary arteries with a normal left ventriculogram. The patient had gradually recovered. Ragunanthanan $\mathrm{Et} \mathrm{al}^{8}$ reported a 60 year old male who developed inferior wall myocardial infarction following Bee Sting.

The pathophysiology explaining the associated myocardial infarction is unknown. Hypotension caused by anaphylaxis may certainly induce myocardial ischaemia; however, this was not documented in our case. In acute myocardial infarction after hymenoptera stings, it has been suggested that vasoconstriction secondary to mediators released after the sting, aggravated by exogenous adrenaline, and platelet aggregation contributes to myocardial ischaemia. ${ }^{9}$ Bee venom itself contains histamine, thromboxane, leucotrienes, and other vasoactive inflammatory mediators. In our patient, we postulate that the systemic immune mediated reaction to the bee sting caused vasoconstriction and a prothrombotic state with subsequent ischaemia leading to myocardial infarction.

\section{Conclusion}

Cardiac complications can accompany bee sting. Intensive supportive treatment in intensive or coronary care facilities with administration of drugs to treat complications early in the course of the illness will improve the outcome.

\section{References}

[1]. Ewan PW. ABC of allergies: venom allergy. BMJ 1998;316:1365-1368.

[2]. Cannon RO, Bonow RO, Bacharach SL et al. Left ventricular dysfunction in patients with angina pectoris, normal epicardial coronary arteries and abnormal vasodilator reserve. Circulation 1985;71:218-26.

[3]. Massing JL, Bentz MH, Schlesser P, Dumitru C, Louis JP. Myocardial infarction following a bee sting. Apropos of a case and review of the literature. Ann Cardiol Angeiol (Paris) 1997;46:311-5.

[4]. Mathieu Vallaa, Frédéric Moulin, Michael Angioiaa, Laurent Grobena, Nicolas Sadoulaa, Etienne Aliot. Myocardial infarction in a 45-year-old man following an anaphylactic reaction to a wasp sting. Int J Cardiol 2009;134:384-392.

[5]. Ceyhan C, Ercan E et al. Myocardial infarction following a bee sting, Int J Cardiol 2001;80:251-3.

[6]. MD Harold, D Levine et al, Acute myocardial infarction following wasp sting*: Report of two cases and critical survey of the literature. American Heart Journal 1976;91:365-374.

[7]. Sofiene Rekik MD, Stephane Andrieu MD et al ST Elevation Myocardial Infarction with No Structural Lesions after a Wasp Sting. Journal of Emergency Medicine 2009;42:73-75.

[8]. Ragunanthanan Et al, Journal of the Association of Physicians of india • Vol 62 published on August, 2014:738-740

[9]. Crawley F, Schon F, Brown MM. Cerebral infarction: a rare complication of wasp sting. J Neurol Neurosurg Psychiatry 1999;66:550-551. 